\title{
Subject-specific calibration of neuromuscular parameters enables neuromusculoskeletal models to estimate physiologically plausible hip joint contact forces in healthy adults
}

Authors: Hoa X. Hoang ${ }^{1,2}$, Claudio Pizzolato ${ }^{1,2}$, Laura E. Diamond ${ }^{1,2}$, David G. Lloyd ${ }^{1,2}$

\author{
${ }^{1}$ School of Allied Health Sciences, Griffith University, Gold Coast, 4222, Australia \\ ${ }^{2}$ Menzies Health Institute Queensland, Griffith University, Gold Coast, 4222, Australia
}

\begin{abstract}
In-vivo hip joint contact forces (HJCF) can be estimated using computational neuromusculoskeletal (NMS) modelling. However, different neural solutions can result in different HJCF estimations. NMS model predictions are also influenced by the selection of neuromuscular parameters, which are either based on cadaveric data or calibrated to the individual. To date, the best combination of neural solution and parameter calibration to obtain plausible estimations of HJCF have not been identified. The aim of this study was to determine the effect of three electromyography (EMG)-informed neural solution modes (EMG-driven, EMG-hybrid, and EMG-assisted) and static optimisation, each using three
\end{abstract} different parameter calibrations (uncalibrated, minimise joint moments error, and minimise joint moments error and peak HJCF), on the estimation of HJCF in a healthy population $(n=23)$ during walking. When compared to existing in-vivo data, the EMG-assisted mode and static optimisation produced the most physiologically plausible HJCF when using a NMS model calibrated to minimise joint moments error and peak HJCF. EMG-assisted mode produced first and second peaks of 3.55 times body weight (BW) and 3.97 BW during 
walking; static optimisation produced 3.75 BW and 4.19 BW, respectively. However, compared to static optimisation, EMG-assisted mode generated muscle excitations closer to recorded EMG signals (average across hip muscles $R^{2}=0.60 \pm 0.37$ versus $R^{2}=0.12 \pm 0.14$ ). Findings suggest that the EMG-assisted mode combined with minimise joint moments error and peak HJCF calibration is preferable for the estimation of HJCF and generation of realistic load distribution across muscles.

\section{Introduction}

Abnormal joint contact forces during walking are considered a primary mechanical risk factor for initiation and progression of osteoarthritis (OA) (Andriacchi and Mündermann, 2006; Felson, 2013; Loeser et al., 2012; Radin et al., 1972). Although in-vivo joint contact forces have been measured in the knee (Fregly et al., 2012) and hip (Bergmann et al., 2016; Brand et al., 1994) with instrumented implants, direct measurement in the native joint is invasive. Computational neuromusculoskeletal (NMS) modelling is a feasible, non-invasive alternative to estimate joint contact forces, although it is unclear which computational modelling approach is best (i.e. electromyography (EMG)-informed or static optimisation). Identification of a viable and accurate computational approach to estimate hip joint contact forces (HJCF) is critical for the advancement of treatments for individuals with hip OA.

Computational NMS models, which are mathematical representations of the anatomy and physiology of an individual, can estimate joint contact forces in the native joints during various motor tasks (Gerus et al., 2013; Saxby et al., 2016; van den Bogert et al., 1999). However, the musculoskeletal system is highly redundant, whereby infinite 
combinations of muscle forces result in the same external joint kinematics and kinetics (Crowninshield and Brand, 1981). Neural solutions are computational algorithms to solve for this redundancy and can be categorised as either an optimisation or EMG-informed approach. Static or dynamic optimisation solve for each muscle's neural drive by minimising an objective criterion, e.g. muscle stresses or muscle activations (Anderson and Pandy, 2001; Crowninshield and Brand, 1981). However, these optimisation criteria have limited physiological basis and assume identical neuromuscular control strategies across individuals. This assumption has dramatic implications for the viability of the optimisation approach as muscle activations can vary across tasks and individuals, even when joint angles and joint moments are the same (Buchanan and Lloyd, 1995; Tax et al., 1990). EMG-informed approaches make no assumptions regarding optimisation criteria, rather using EMG signals to determine muscle activation patterns to account for each individual's unique neural solution (Lloyd and Buchanan, 1996; Thelen et al., 1994).

EMG-informed modelling has three neural solution modes: EMG-driven, EMG-hybrid, and EMG-assisted. The EMG-driven mode uses only recorded EMG signals to drive the model in an open-loop predictive system (Lloyd and Besier, 2003). However, many muscles important for hip function (i.e. iliacus and psoas) are located below the surface, making the acquisition of EMG signals challenging. The EMG-hybrid mode uses static optimisation to synthesise EMG signals that were not acquired experimentally (Sartori et al., 2014). Although EMG-driven and EMG-hybrid approaches are advantageous compared to static optimisation methods, the quality of EMG signals can be affected by crosstalk, poor electrode placement, and skin impedance (Farina and Negro, 2012). To mitigate the effect 
of measurement errors, the EMG-assisted mode uses recorded EMG signals as inputs and allows optimisation to adjust excitations to improve joint moment tracking.

EMG-informed models are calibrated to the individual, whereby an optimisation procedure (i.e. calibration) estimates a set of neuromuscular parameters (i.e. tendon slack length, optimal fibre length, maximum isometric force, non-linear shape factor, EMG-toactivation recursive filter coefficients) to best match experimental and estimated joint moments (Lloyd and Besier, 2003; Pizzolato et al., 2015). Unfortunately, this optimisation does not guarantee accurate joint contact force estimations (Fregly et al., 2012). Performing a calibration where both peak joint contact forces and the error between experimental and estimated joint moments are minimised has been shown to improve estimations and produce more physiologically plausible knee joint contact forces (KJCF) (i.e. more comparable results to in-vivo data while best matching all experimental data) (Gerus et al., 2013). Conceivably, the prediction of HJCF could also be improved by minimising HJCF peaks and joint moment errors during the calibration procedure.

Although EMG-informed methods are now well established at the knee (Gerus et al., 2013; Konrath et al., 2016; Lloyd and Besier, 2003; Manal and Buchanan, 2013; Saxby et al., 2016), their application to the hip is still limited (Fernandez et al., 2014; Pizzolato et al., 2015). Thus, it is unclear how EMG-informed approaches compare with static optimisation when modelling the hip joint and if any calibration procedure is favourable for estimating physiologically plausible HJCF. The aims of this study were: (1) to determine if EMGinformed modelling is a viable method to estimate HJCF compared to static optimisation; and (2) to identify the effects of three different parameter calibrations (uncalibrated, minimise joint moments error, and minimise joint moments error and peak HJCF) on the 
prediction of HJCF. We hypothesised that the EMG-assisted mode of EMG-informed modelling would produce the best tracking of joint moments and muscle excitations, and lower HJCF when compared to other EMG-informed modes and static optimisation. Furthermore, we hypothesised that minimising peak HJCF in the calibration will produce models that estimate more physiologically plausible HJCF compared to those produced with other calibrations.

\section{Methods}

This study integrated gait analysis data with surface EMG signals for use in EMGinformed modelling to estimate muscle forces. The resulting muscle forces were used to estimate HJCF. The Griffith University Human Research Ethics Committee granted ethical approval, and all participants gave their written informed consent prior to starting experimentation. The 23 participants (age $60.1 \pm 7.6$ years, body mass $69.7 \pm 8.9 \mathrm{~kg}$, height $1.67 \pm 0.08 \mathrm{~m}$ ) were healthy and included if their: (i) radiological hip joint space width $>3 \mathrm{~mm}$ in both hips; (ii) Kellgren-Lawrence grades $\leq 1$ (Kellgren and Lawrence, 1957); and (iii) Harris Hip Score $>95$ points (Mahomed et al., 2001).

Retro-reflective skin surface markers were placed on each participant following the Griffith marker set (Graham et al., 2014). A 12-camera VICON MX motion analysis system (Vicon, Oxford, UK) sampling at $200 \mathrm{~Hz}$ acquired marker trajectories. Ground reaction forces (GRF) were measured using two force plates (Kistler, Amherst, USA) sampling at $1000 \mathrm{~Hz}$. EMG signals from 16 muscles (Sartori et al., 2014) of a single randomly-selected leg were recorded at $1000 \mathrm{~Hz}$ using a wireless EMG system (Cometa, Milan, Italy). Surface EMG electrodes were placed in accordance with SENIAM guidelines (Hermens et al., 2000) for the 
following muscles: biceps femoris (BF), semimembranosus (SM), gluteus maximus (GMAX), gluteus medius (GMED), adductor magnus (AM), rectus femoris (RF), sartorius (SAR), vastus lateralis (VL), vastus medialis (VM), tensor fascia latae (TFL), gracilis (GRA), medial gastrocnemius $(\mathrm{GM})$, lateral gastrocnemius $(\mathrm{GL})$, peroneals $(\mathrm{PER})$, soleus $(\mathrm{SOL})$, and tibialis anterior (TA).

Participants performed ten walking trials barefoot at a self-selected speed $(1.20 \pm 0.11 \mathrm{~m} / \mathrm{s})$ over a $10-$ metre walkway. To elicit maximum muscle contractions, particpants performed a maximal vertical counter movement jump (Padulo et al., 2013) and a set of maximum isometric voluntary contractions (MVC) (Pizzolato et al., 2017) on a dynamometer (Biodex Medical Systems, Shirley, NY, USA) for hip and knee flexion/extension (FE), hip adduction/abduction (AA), and ankle dorsi/plantar flexion (DPF). MVC for muscles crossing the hip were acquired in standing, with the leg in $0^{\circ}$ hip abduction, hip flexion, knee flexion, and ankle plantarflexion. MVC for muscles crossing the knee and ankle were acquired in a seated position at $80^{\circ}$ hip flexion, $30^{\circ}$ knee flexion, and $15^{\circ}$ ankle plantarflexion.

\section{Experimental data processing}

Data was processed using MOtoNMS (Mantoan et al., 2015). Marker trajectories and GRF were low-pass filtered using a zero-lag, $2^{\text {nd }}$ order, $6 \mathrm{~Hz}$ Butterworth filter (Robertson and Dowling, 2003). Hip joint centres were defined using the Harrington regression equation (Harrington et al., 2007); knee joint centres were defined as the midpoint of the lateral and medial femoral condyle markers; ankle joint centres were defined as the midpoint between the medial and lateral malleoli markers with an offset (0.027 $x$ shank length) (Bruening et al., 2008). EMG signals were band-pass filtered $(30-300 \mathrm{~Hz})$, full wave rectified, and low-pass 
filtered at $6 \mathrm{~Hz}$ (Lloyd and Besier, 2003). Muscle excitations were calculated from the filtered EMG signals amplitude-normalised to the maximum processed EMG value recorded across all trial types (dynamometer, walking and jump trials), with the normalisation trial type varying across muscles and participants.

A generic OpenSim model (gait2392) was linearly scaled to each participant in OpenSim version 3.2 (Delp et al., 2007) per Kainz et al. (2017). The model had the following degrees-of-freedom (DOF): hip and knee FE, hip AA, hip internal/external rotation (IER), and ankle DPF. The model was simplified to include 34 muscle-tendon units (MTUs) actuating one side of the lower limb (Sartori et al., 2012). The optimal fibre length and tendon slack length were morphometrically scaled using an optimisation technique (Modenese et al., 2016). This process preserved the dimensionless muscle fibre and tendon operating curves after OpenSim model scaling. The final scaled model was used in the EMG-informed modelling pipeline.

OpenSim was employed to calculate joint angles and moments, and MTU kinematics (i.e. muscle lengths and moment arms). Inverse kinematics and inverse dynamics were respectively used to calculate joint angles and moments for each walking trial. The muscle analysis tool was used to calculate MTU lengths and moment arms, which were visually checked to be continuous. The OpenSim data and EMG-derived muscle excitations were used as inputs to the EMG-informed modelling package, Calibrated EMG-informed Neuromusculoskeletal Modelling Toolbox (CEINMS) (Pizzolato et al., 2015). CEINMS was used to calculate muscle forces, which was then used to estimate the HJCF via the Joint Reaction analysis (Opensim application programming interface). The Joint Reaction analysis 
calculated the HJCF using a force balance on each rigid body starting from the most distal and moving proximally (Steele et al., 2012).

CEINMS operates in two steps: first in the calibration process and then in predictive execution mode. In CEINMS, the neural activation was represented by a critically-damped linear second-order differential system (Lloyd and Besier, 2003):

$$
u(t)=\alpha e(t-d)-\left(C_{1}+C_{2}\right) u(t-1)-C_{1} C_{2} u(t-2)
$$

where $u(t)$ is the neural activation, $a$ is the muscle gain, $e(t)$ is a muscle excitation at time $t, d$ is the electromechanical delay, and $C_{1}$ and $C_{2}$ are the recursive coefficients. The relationship between the neural activation and muscle activation is non-linear and described by Lloyd and Besier (2003) as:

$$
a(t)=\frac{e^{A u(t)}-1}{e^{A}-1}
$$

where $a(t)$ is the muscle activation; and $A$ is the non-linear shape factor, constraint to be between -3 and 0 , with 0 representing a linear EMG-to-force relationship and negative values a non-linear relationship. Musculotendon dynamics were solved using a Winjingaarden-Dekker-Brent optimisation routine (Brent, 1973) implemented in CEINMS (Pizzolato et al., 2015). As per previous studies in EMG-informed modelling (Gerus et al., 2013; Lloyd and Besier, 2003; Pizzolato et al., 2015; Sartori et al., 2012), a simulated annealing optimisation (Corana et al., 1987) was used to adjust $A, C_{1}, C_{2}$, tendon slack length, optimal fibre length, and maximum isometric force, which were bound to be within physiological range (Table 1). 
Two calibration methods were implemented. The first was the default calibration method (minMoments) in EMG-driven modelling (Lloyd and Besier, 2003; Sartori et al., 2012). The objective function being minimised for minMoments calibration was:

$$
f_{\text {minMoments }}=\sum_{t}^{N_{\text {trials }}} \sum_{d}^{N_{D O F}} E_{t, d}
$$

where the error term, $E_{t, d}$, is the sum of the squared differences between the predicted joint moments and the experimentally measured joint moments for each degree of freedom and trial in the calibration normalised by trial variance (Pizzolato et al., 2015). The experimental joint moments were acquired from previously calculated joint moments in OpenSim using inverse dynamics. The joint moments included in the calibration were hip and knee FE, hip AA, and ankle DPF (Sartori et al., 2012). The second calibration method includes an additional term in the cost function to minimise the peak HJCF (minHJCF). The objective function being minimised for minHJCF calibration was:

$$
f_{\text {min } H C F}=\sum_{t}^{N_{\text {trials }}} \sum_{d}^{N_{D O F}} E_{t, d}+w H J C F_{\text {peak }}
$$

where $w$ is a weight factor and $H J C F_{\text {peak }}$ is the peak HJCF magnitude for all the trials used in the calibration. To ensure the calibration did not favour minimisation $H J C F_{\text {peak }}$ (units in newtons) compared to tracking joint moments, $w$ was selected so that the $H J C F_{\text {peak }}$ error term had the same order of magnitude to the $E_{t, d}$ error term. The 16 experimental muscle excitations were allocated to the 34 MTUs in CEINMS according to Sartori et al. (2012). Four walking trials were used during calibration. Finally, an uncalibrated model (unCal) was created for comparison, resulting in three calibration models (i.e. unCal, minMoments, and 
minHJCF) per participant with different neuromuscular parameters. The unCal model had muscle parameters previously scaled in OpenSim while $A, C_{1}$, and $C_{2}$ were set to initial values (Table 1)

After calibration, muscle forces were estimated for each calibration model with the remaining six walking trials using each of the neural solution modes in CEINMS using established methods from Sartori et al. (2014) and Pizzolato et al. (2015): "EMG-driven", "EMG-hybrid", "EMG-assisted", and "Static Optimisation". All neural solutions modes solve the muscle forces frame-by-frame using non-linear least squares optimisation (Pizzolato et al., 2015). For the EMG-assisted mode, the objective function being minimised was:

$$
f_{E M G-\text { assisted }}=\alpha E_{\text {Moment }}+\beta E_{\text {sumEXC }}+\gamma E_{E M G}
$$

where $E_{\text {Мом }}$ is the sum of the squared differences between experimental and predicted joint moments, $E_{S U m E X C}$ is the sum of squared excitations for all $34 \mathrm{MTUs}, E_{E M G}$ is the sum of the differences between experimental and adjusted EMG excitations for the 32 MTUs with EMG recordings, and $(\alpha, b, \gamma)$ are positive weighting coefficients. With $\alpha$ set to $1, b$ and $\gamma$ weightings (Pizzolato et al., 2015) were optimised to balance between joint moment error $\left(E_{M O M}\right)$ and muscle excitation errors $\left(E_{E M G}\right)$ (Sartori et al., 2014). For static optimisation, the standard objective function to minimise the sum of the excitation squared was used to estimate muscle forces (Pizzolato et al., 2015).

\section{Statistical analysis}

Coefficient of determination $\left(R^{2}\right)$ and root-mean-square error (RMSE) were calculated for the predicted and experimental hip FE moments for each calibration and neural solution mode. First and second peaks of the resultant HJCF (HJCF peak1, HJCF peak2) 
were calculated for each calibration and neural solution mode. In-vivo HJCF data were included for comparison $(n=10$, age $=56.9 \pm 5.2 \mathrm{yr}$, body mass $=88.7 \pm 12.4 \mathrm{~kg}$, height $=$ 174.0 $\pm 5.9 \mathrm{~cm}$, walking speed $=1.1 \mathrm{~m} / \mathrm{s}$ (range 1.0-1.3m $/ \mathrm{s})$ (Bergmann et al., 2016). The $\mathrm{R}^{2}$ and RMSE were calculated for predicted muscle excitations using the EMG-assisted mode and static optimisation. The EMG-driven and EMG-hybrid modes were not used in this analysis since these directly used recorded EMG signals as inputs and thus produce $R^{2}=1$ and RMSE $=0$. For each neural solution mode, a one-way repeated measures ANOVA $(p<0.05)$ was used to compare hip FE moments $\mathrm{R}^{2}$, HJCF peaks, and hip muscle excitations $\mathrm{R}^{2}$ produced from all calibrations. When significant differences were detected $(p<0.05)$, a Bonferroni correction was applied (IBM SPSS Statistics 24).

\section{Results}

The hip FE moment $\mathrm{R}^{2}$ was higher in both minHJCF $(p<0.001)$ and minMoments $(p<0.001)$ calibrations for all EMG-informed solution modes when compared to unCal (Figure 1; Table 2). The minMoments calibration had higher $\mathrm{R}^{2}$ and lower RMSE than minHJCF calibration for EMG-driven and EMG-hybrid modes $(p<0.001) . \mathrm{R}^{2}$ was lower with calibration for static optimisation; however, $\mathrm{R}^{2}$ was not significantly different between the calibrations with all values close to 1 . EMG-assisted mode and static optimisation produced high $R^{2}(>0.97)$ and low RMSE $(<0.12 \mathrm{Nm} / \mathrm{kg})$ for all calibrations.

Calibrating with minHJCF produced significantly lower HJCF $_{\text {peak1 }}$ for all EMG-informed modes when compared to the unCal and minMoments calibration (Figure 2; Table 3; Table 4). Calibrating with minMoments did not produce significantly different $\mathrm{HJCF}_{\text {peak1 }}$ when compared with unCal for all EMG-informed modes. The minMoments in static optimisation produced significantly higher HJCF peak1 than unCal $(p<0.001)$ and minHJCF $(p<0.001)$. Using 
static optimisation with minHJCF produced lower, albeit not significant, HJCF $_{\text {peak1 } 1}$ compared to unCal. This pattern was similar for $\mathrm{HJCF}_{\text {peak2 }}$ using EMG-driven and EMG-hybrid modes where unCal and minHJCF were not significantly different, but were both significantly lower than minMoments calibration $(p<0.001)$. Calibrating with minHJCF for EMG-assisted and static optimisation produced significantly lower $\mathrm{HJCF}_{\text {peak2 }}$ when compared to unCal $(p<0.001)$ and minMoments calibrations $(p<0.001)$.

For the EMG-assisted mode, minHJCF calibration produced higher $\mathrm{R}^{2}$ for all hip muscles excitations except gluteus maximus, rectus femoris, and gracilis (Figure 3; Table 4). For static optimisation, there was a systemic increase in $\mathrm{R}^{2}$ with minHJCF calibration, but only the lateral hamstring produced an $\mathrm{R}^{2}$ value $>0.5$. Calibration lowered the standard deviation (STD) for all muscles compared to unCal in EMG-assisted mode, though calibration increased the STD for static optimisation. RMSE for all hip muscles excitations were low in the EMG-assisted mode $(<0.2)$.

\section{Discussion}

The aim of this study was to determine if EMG-informed modelling is suitable to estimate HJCF and assess the effects of different calibrations on HJCF estimates. A calibration that minimised joint moments error and peak HJCF (minHJCF) resulted in lower HJCF estimates for all neural solution modes. Further, EMG-assisted mode was able to match experimental moments and estimate physiologically plausible HJCF despite lacking recorded EMG signals from the deep hip muscles.

EMG-assisted and static optimisation modes correctly estimated hip FE moments (Figure 1) as well as joint moments in the other DOF (Table 2). EMG-assisted mode can effectively track joint moments because it allows for adjustment of EMG signals to match 
joint moments; static optimisation matches joint moments by design. Conversely, EMG-

driven and EMG-hybrid modes were unable to correctly estimate hip FE moments (Figure 1).

Though the EMG-driven mode was predicted to perform poorly given the absence of EMG signals from iliacus and psoas major, the poor prediction of the EMG-hybrid mode was surprising. This was most evident for the hip extension moment in early stance, and thus may relate to low signal to noise in surface EMG signals for the gluteus maximus, a primary contributor to hip extension, which was not adjusted by the EMG-hybrid mode.

The minMoments calibration improved the estimation of hip FE moments for EMGdriven and EMG-hybrid. However, it did not further improve EMG-assisted and static optimisation joint moment tracking when compared to the unCal calibration (Figure 1; Table 2). Conversely, the minHJCF calibration increased the error between experimental and predicted hip FE for all modes, but also reduced the HJCF peaks for all the modes (Figure 2).

Among the neural solution modes tested, EMG-assisted and static optimisation, in conjunction with minHJCF calibration, were the best neural solution modes for estimating HJCF and produced comparable results for our healthy cohort (Figure 2; Table 3). This suggests that an additional objective function such as minimising peak HJCF during the calibration process is needed to adjust neuromuscular parameters that best track joint moments and muscle excitations to produce physiologically plausible HJCF. Our estimated HJCF using EMG-assisted and static optimisation were generally larger than the in-vivo data (Bergmann et al., 2016). However, our healthy participants, with native joints, cannot be directly compared to individuals with a hip implant, who generally have weaker muscles and altered motor control (Long et al., 1993; Shih et al., 1994). These neuromuscular deficits may explain the differences between our HJCF estimates and the in-vivo data, given HJCF 
depend on both muscle and external forces (Herzog et al., 2003). With minHJCF calibration, EMG-driven and EMG-hybrid modes produced HJCF closer to that of Bergmann's in-vivo dataset (2016). However, neither EMG-driven nor EMG-hybrid are suitable approaches to estimate HJCF given their inability to match external hip joint moments (Figure 1).

Although EMG-assisted and static optimisation predicted similar HJCF for our cohort, EMG-assisted was superior for tracking hip muscle excitations, resulting in higher $\mathrm{R}^{2}$ and lower RMSE for all hip muscles (Figure 3; Table 5). This suggests a more plausible distribution of load across hip muscles, suggesting the EMG-assisted mode may be most suitable for hip joint investigations. It was expected that calibration would improve tracking of muscle excitations for EMG-assisted; however, calibration also improved muscle excitation tracking for static optimisation. The gluteus maximus was the only major contributor to HJCF (Correa et al., 2010) that had a decrease in $\mathrm{R}^{2}$ muscle excitations after calibration. This may relate to low quality recordings from surface electrodes applied in an area with adipose tissue.

Collection of experimental EMG signals and model calibration are time intensive, but essential for EMG-informed modelling. Static optimisation is often used due to its simplicity; however, the EMG-assisted mode combined with minHJCF calibration presented here proved better for tracking hip muscle excitations. This may have important implications for people with hip OA, who often exhibit hip muscle weakness (Loureiro et al., 2013) and altered hip muscle activation patterns (Rutherford et al., 2015; Sims et al., 2002). Wesseling et al. (2016) demonstrated that HJCF minimisation had no effect on overall prediction of HJCF. However, their HJCF minimisation was performed within the static optimisation criteria, thus influencing the estimation of muscle activation rather than neuromuscular 
parameters of the NMS model. This differs from our implementation, which influenced neuromuscular parameters in the model and generated lower prediction of HJCF peaks.

Previous studies involving people with instrumented knee implants (Fregly et al., 2012) have also shown that calibrating the NMS model to the individual is essential in estimating accurate KJCF (Gerus et al., 2013; Serrancolí et al., 2016). Furthermore, tracking experimental KJCF measurements (Serrancolí et al., 2016) or minimising peak KJCF (Gerus et al., 2013) were additional calibration criteria that successfully improved KJCF estimates. Although lower HJCF peaks were expected by imposing an objective function to minimise peak HJCF during calibration, our results show that tracking of joint moments and muscle excitations also improved with this calibration. Our results from the hip joint corroborate and extend previous findings on the importance of tuning musculoskeletal parameters to the individual. Furthermore, our findings also suggest that using external joint moments tracking alone to calibrate the model's neuromuscular parameters is insufficient to attain physiologically plausible joint contact forces (Figure 2).

There are several limitations to this study. The absence of in-vivo HJCF data for a healthy population hinders direct validation of our results. Further validation of EMGinformed modelling to estimate HJCF will be feasible if EMG signals become available with in-vivo HJCF data, similar to those available at the knee (Fregly et al., 2012). We have provided a comparison to instrumented HJCF data from people with hip replacement (Bergmann et al., 2016), which is the only currently available; however, EMG data was not available for comparison. The challenges in recording muscle excitations from the iliacus and psoas major is also a limitation of estimating muscle forces at the hip using EMG-informed modelling. As such, we are unable to determine how well our models predict these muscle 
excitations. We used walking trials to calibrate the NMS model as they were the only task available in this study. Results may be different if additional tasks were included in the calibration (Falisse et al., 2017). However, different to previous studies (Falisse et al., 2017; Gerus et al., 2013, Lloyd and Besier, 2003) we did not use a single joint with single DOF calibration. Rather, our calibration used hip, knee, and ankle joints with multiple degrees of freedom, which has been shown to produce superior results (Sartori et al., 2012). Nevertheless, future evaluations should investigate the effect of different motor tasks in the calibration on HJCF estimates. We used an objective function to minimise the peak HJCF in the calibration; however, other objective functions should be explored in future studies. The anatomical model used was generic and linearly scaled. This could introduce errors in the muscle lines of action and moment arms, which in turn affect the resulting HJCF. Though this is commonly done in NMS modelling (Graham et al., 2016; Kainz et al., 2016; Steele et al., 2012), future investigation should include imaging data to create models with subjectspecific geometries (Gerus et al., 2013; Wesseling et al., 2016) and joint kinematics (Brito da Luz et al., 2017). Subject-specific imaging data will also improve hip joint centre location calculations, an important parameter in estimating HJCF (Lenaerts et al., 2009). Finally, the current contact model for the hip is a simple point contact; more complex models, such as elastic foundation or finite element modelling, could be used to better represent the acetabulum morphology (Fernandez et al., 2014).

This study estimated HJCF during walking in a healthy adult population using different calibration cost functions and different neural solution modes in EMG-informed modelling, and compared these with static optimisation. Findings suggest the calibration cost function is a major factor in estimating physiologically plausible HJCF and that minHJCF 
calibration and EMG-assisted mode should be used when investigating the hip joint.

Conversely, EMG-driven may not be appropriate for investigations of the hip joint, as it cannot account for iliacus and psoas major muscles in the absence of direct recordings. Although our results show similar HJCF prediction with EMG-assisted and static optimisation, EMG-assisted generated a more realistic load distribution across the hip muscles because it estimated muscle excitations closer to measured muscle excitations. Consequently, EMG-assisted mode may be most appropriate for investigations of individuals with hip pathology who are likely to have different motor control strategies.

\section{Acknowledgments}

This work was funded by a postgraduate scholarship from Griffith University and Menzies Health Institute Queensland and by a Griffith University Strategic Investments Grant for the project. The authors would like to thank Prof Rod Barrett, Dr Peter Mills, Dr Luca Modenese, Dr Aderson Loureiro, Dr Maria Constantinou, Jeremy Higgs, and all participants for their support of the project. We would also like to thank A/Prof Monica Reggiani and Dr Alice Mantoan for the development of MOtoNMS.

\section{Conflict of interest statement}

The authors declare no conflicts of interest.

\section{References}

Anderson, F.C., Pandy, M.G., 2001. Dynamic Optimization of Human Walking. J. Biomech. Eng. 123, 381-390. https://doi.org/10.1115/1.1392310

Andriacchi, T.P., Mündermann, A., 2006. The role of ambulatory mechanics in the initiation and progression of knee osteoarthritis. Curr. Opin. Rheumatol. Sept. 2006 18, 514518. https://doi.org/10.1097/01.bor.0000240365.16842.4e

Bergmann, G., Bender, A., Dymke, J., Duda, G., Damm, P., 2016. Standardized Loads Acting in Hip Implants. PLOS ONE 11, e0155612.

https://doi.org/10.1371/journal.pone.0155612 
Brand, R.A., Pedersen, D.R., Davy, D.T., Kotzar, G.M., Heiple, K.G., Goldberg, V.M., 1994. Comparison of hip force calculations and measurements in the same patient. J. Arthroplasty 9, 45-51. https://doi.org/10.1016/0883-5403(94)90136-8

Brent, R., 1973. Some Efficient Algorithms for Solving Systems of Nonlinear Equations. SIAM J. Numer. Anal. 10, 327-344. https://doi.org/10.1137/0710031

Brito da Luz, S., Modenese, L., Sancisi, N., Mills, P.M., Kennedy, B., Beck, B.R., Lloyd, D.G., 2017. Feasibility of using MRIs to create subject-specific parallel-mechanism joint models. J. Biomech. 53, 45-55. https://doi.org/10.1016/j.jbiomech.2016.12.018

Bruening, D.A., Crewe, A.N., Buczek, F.L., 2008. A simple, anatomically based correction to the conventional ankle joint center. Clin. Biomech. 23, 1299-1302. https://doi.org/10.1016/j.clinbiomech.2008.08.005

Buchanan, T.S., Lloyd, D.G., 1995. Muscle activity is different for humans performing static tasks which require force control and position control. Neurosci Lett 194, 61-4.

Corana, A., Marchesi, M., Martini, C., Ridella, S., 1987. Minimizing Multimodal Functions of Continuous Variables with the "Simulated Annealing" algorithmCorrigenda for This Article is Available Here. ACM Trans Math Softw 13, 262-280. https://doi.org/10.1145/29380.29864

Correa, T.A., Crossley, K.M., Kim, H.J., Pandy, M.G., 2010. Contributions of individual muscles to hip joint contact force in normal walking. J. Biomech. 43, 1618-1622. https://doi.org/10.1016/j.jbiomech.2010.02.008

Crowninshield, R.D., Brand, R.A., 1981. The Prediction of Forces in Joint Structures: Distribution of Intersegmental Resultants. Exerc. Sport Sci. Rev. 9, 159-182.

Delp, S.L., Anderson, F.C., Arnold, A.S., Loan, P., Habib, A., John, C.T., Guendelman, E., Thelen, D.G., 2007. OpenSim: open-source software to create and analyze dynamic simulations of movement. IEEE Trans. Biomed. Eng. 54, 1940-1950. https://doi.org/10.1109/TBME.2007.901024

Falisse, A., Van Rossom, S., Jonkers, I., De Groote, F., 2017. EMG-Driven Optimal Estimation of Subject-SPECIFIC Hill Model Muscle-Tendon Parameters of the Knee Joint Actuators. IEEE Trans. Biomed. Eng. 64, 2253-2262. https://doi.org/10.1109/TBME.2016.2630009

Farina, D., Negro, F., 2012. Accessing the Neural Drive to Muscle and Translation to Neurorehabilitation Technologies. Biomed. Eng. IEEE Rev. In 5, 3-14. https://doi.org/10.1109/RBME.2012.2183586

Felson, D.T., 2013. Osteoarthritis as a disease of mechanics. Osteoarthritis Cartilage 21, 1015. https://doi.org/10.1016/j.joca.2012.09.012

Fernandez, J., Sartori, M., Lloyd, D., Munro, J., Shim, V., 2014. Bone remodelling in the natural acetabulum is influenced by muscle force-induced bone stress. Int. J. Numer. Methods Biomed. Eng. 30, 28-41. https://doi.org/10.1002/cnm.2586

Fregly, B.J., Besier, T.F., Lloyd, D.G., Delp, S.L., Banks, S.A., Pandy, M.G., D'Lima, D.D., 2012. Grand challenge competition to predict in vivo knee loads. J. Orthop. Res. 30, 503513. https://doi.org/10.1002/jor.22023

Gerus, P., Sartori, M., Besier, T.F., Fregly, B.J., Delp, S.L., Banks, S.A., Pandy, M.G., D'Lima, D.D., Lloyd, D.G., 2013. Subject-specific knee joint geometry improves predictions of medial tibiofemoral contact forces. J. Biomech. 46, 2778-2786. https://doi.org/10.1016/j.jbiomech.2013.09.005 
Graham, D.F., Carty, C.P., Lloyd, D.G., Lichtwark, G.A., Barrett, R.S., 2014. Muscle contributions to recovery from forward loss of balance by stepping. J. Biomech. 47, 667-674. https://doi.org/10.1016/j.jbiomech.2013.11.047

Graham, D.F., Modenese, L., Trewartha, G., Carty, C.P., Constantinou, M., Lloyd, D.G., Barrett, R.S., 2016. Hip joint contact loads in older adults during recovery from forward loss of balance by stepping. J. Biomech. 49, 2619-2624. https://doi.org/10.1016/j.jbiomech.2016.05.033

Harrington, M.E., Zavatsky, A.B., Lawson, S.E.M., Yuan, Z., Theologis, T.N., 2007. Prediction of the hip joint centre in adults, children, and patients with cerebral palsy based on magnetic resonance imaging. J. Biomech. 40, 595-602. https://doi.org/10.1016/j.jbiomech.2006.02.003

Hermens, H.J., Freriks, B., Disselhorst-Klug, C., Rau, G., 2000. Development of recommendations for SEMG sensors and sensor placement procedures. J. Electromyogr. Kinesiol. 10, 361-374. https://doi.org/10.1016/S1050-6411(00)000274

Herzog, W., Clark, A., Wu, J., 2003. Resultant and local loading in models of joint disease. Arthritis Care Res. 49, 239-247. https://doi.org/10.1002/art.11004

Kainz, H., Hoang, H., Stockton, C., Boyd, R.R., Lloyd, D.G., Carty, C.P., 2017. Accuracy and Reliability of Marker Based Approaches to Scale the Pelvis, Thigh and Shank Segments in Musculoskeletal Models. J. Appl. Biomech. 1-21. https://doi.org/10.1123/jab.2016-0282

Kainz, H., Modenese, L., Lloyd, D.G., Maine, S., Walsh, H.P.J., Carty, C.P., 2016. Joint kinematic calculation based on clinical direct kinematic versus inverse kinematic gait models. J. Biomech. 49, 1658-1669. https://doi.org/10.1016/j.jbiomech.2016.03.052

Kellgren, J.H., Lawrence, J.S., 1957. Radiological Assessment of Osteo-Arthrosis. Ann. Rheum. Dis. 16, 494-502. https://doi.org/10.1136/ard.16.4.494

Konrath, J.M., Vertullo, C.J., Kennedy, B.A., Bush, H.S., Barrett, R.S., Lloyd, D.G., 2016. Morphologic Characteristics and Strength of the Hamstring Muscles Remain Altered at 2 Years After Use of a Hamstring Tendon Graft in Anterior Cruciate Ligament Reconstruction. Am. J. Sports Med. 44, 2589-2598. https://doi.org/10.1177/0363546516651441

Lenaerts, G., Bartels, W., Gelaude, F., Mulier, M., Spaepen, A., Van der Perre, G., Jonkers, I., 2009. Subject-specific hip geometry and hip joint centre location affects calculated contact forces at the hip during gait. J. Biomech. 42, 1246-1251. https://doi.org/10.1016/j.jbiomech.2009.03.037

Lloyd, D.G., Besier, T.F., 2003. An EMG-driven musculoskeletal model to estimate muscle forces and knee joint moments in vivo. J. Biomech. 36, 765-776. https://doi.org/10.1016/S0021-9290(03)00010-1

Lloyd, D.G., Buchanan, T.S., 1996. A model of load sharing between muscles and soft tissues at the human knee during static tasks. J. Biomech. Eng. 118, 367-376.

Loeser, R.F., Goldring, S.R., Scanzello, C.R., Goldring, M.B., 2012. Osteoarthritis: A Disease of the Joint as an Organ. Arthritis Rheum. 64, 1697-1707. https://doi.org/10.1002/art.34453

Long, W.T., Dorr, L.D., Healy, B., Perry, J., 1993. Functional recovery of noncemented total hip arthroplasty. Clin. Orthop. 73-77. 
Loureiro, A., Mills, P.M., Barrett, R.S., 2013. Muscle weakness in hip osteoarthritis: A systematic review. Arthritis Care Res. 65, 340-352. https://doi.org/10.1002/acr.21806

Mahomed, N.N., Arndt, D.C., McGrory, B.J., Harris, W.H., 2001. The Harris hip score: Comparison of patient self-report with surgeon assessment. J. Arthroplasty 16, 575580. https://doi.org/10.1054/arth.2001.23716

Manal, K., Buchanan, T.S., 2013. An Electromyogram-Driven Musculoskeletal Model of the Knee to Predict in Vivo Joint Contact Forces During Normal and Novel Gait Patterns. J. Biomech. Eng. 135, 0210141-0210147. https://doi.org/10.1115/1.4023457

Mantoan, A., Pizzolato, C., Sartori, M., Sawacha, Z., Cobelli, C., Reggiani, M., 2015. MOtoNMS: A MATLAB toolbox to process motion data for neuromusculoskeletal modeling and simulation. Source Code Biol. Med. 10, 12. https://doi.org/10.1186/s13029-015-0044-4

Modenese, L., Ceseracciu, E., Reggiani, M., Lloyd, D.G., 2016. Estimation of musculotendon parameters for scaled and subject specific musculoskeletal models using an optimization technique. J. Biomech. 49, 141-148. https://doi.org/10.1016/j.jbiomech.2015.11.006

Padulo, J., Tiloca, A., Powell, D., Granatelli, G., Bianco, A., Paoli, A., 2013. EMG amplitude of the biceps femoris during jumping compared to landing movements. SpringerPlus 2 , 520. https://doi.org/10.1186/2193-1801-2-520

Pizzolato, C., Lloyd, D.G., Sartori, M., Ceseracciu, E., Besier, T.F., Fregly, B.J., Reggiani, M., 2015. CEINMS: A toolbox to investigate the influence of different neural control solutions on the prediction of muscle excitation and joint moments during dynamic motor tasks. J. Biomech. 48, 3929-3936.

https://doi.org/10.1016/j.jbiomech.2015.09.021

Pizzolato, C., Reggiani, M., Saxby, D.J., Ceseracciu, E., Modenese, L., Lloyd, D.G., 2017. Biofeedback for gait retraining based on real-time estimation of tibiofemoral joint contact forces. IEEE Trans. Neural Syst. Rehabil. Eng. PP, 1-1. https://doi.org/10.1109/TNSRE.2017.2683488

Radin, E.L., Paul, I.L., Rose, R.M., 1972. Role of mechanical factors in pathogenesis of primary osteoarthritis. Lancet 1, 519-22.

Robertson, D.G.E., Dowling, J.J., 2003. Design and responses of Butterworth and critically damped digital filters. J. Electromyogr. Kinesiol. Off. J. Int. Soc. Electrophysiol. Kinesiol. 13, 569-573.

Rutherford, D.J., Moreside, J., Wong, I., 2015. Hip joint motion and gluteal muscle activation differences between healthy controls and those with varying degrees of hip osteoarthritis during walking. J. Electromyogr. Kinesiol. 25, 944-950. https://doi.org/10.1016/j.jelekin.2015.10.010

Sartori, M., Farina, D., Lloyd, D.G., 2014. Hybrid neuromusculoskeletal modeling to best track joint moments using a balance between muscle excitations derived from electromyograms and optimization. J. Biomech. 47, 3613-3621. https://doi.org/10.1016/j.jbiomech.2014.10.009

Sartori, M., Reggiani, M., Farina, D., Lloyd, D.G., 2012. EMG-Driven Forward-Dynamic Estimation of Muscle Force and Joint Moment about Multiple Degrees of Freedom in the Human Lower Extremity. PLoS ONE 7, e52618. https://doi.org/10.1371/journal.pone.0052618 
Saxby, D.J., Modenese, L., Bryant, A.L., Gerus, P., Killen, B., Fortin, K., Wrigley, T.V., Bennell, K.L., Cicuttini, F.M., Lloyd, D.G., 2016. Tibiofemoral contact forces during walking, running and sidestepping. Gait Posture 49, 78-85. https://doi.org/10.1016/j.gaitpost.2016.06.014

Serrancolí, G., Kinney, A.L., Fregly, B.J., Font-Llagunes, J.M., 2016. Neuromusculoskeletal Model Calibration Significantly Affects Predicted Knee Contact Forces for Walking. J. Biomech. Eng. 138, 081001-081001. https://doi.org/10.1115/1.4033673

Shih, C.-H., Du, Y.-K., Lin, Y.-H., Wu, C.-C., 1994. Muscular recovery around the hip joint after total hip arthroplasty. Clin. Orthop. 302, 115-120.

Sims, K., Richardson, C., Brauer, S., 2002. Investigation of hip abductor activation in subjects with clinical unilateral hip osteoarthritis. Ann. Rheum. Dis. 61, 687-692. https://doi.org/10.1136/ard.61.8.687

Steele, K.M., DeMers, M.S., Schwartz, M.H., Delp, S.L., 2012. Compressive tibiofemoral force during crouch gait. Gait Posture 35, 556-560. https://doi.org/10.1016/j.gaitpost.2011.11.023

Tax, A.A.M., Denier van der Gon, J.J., Erkelens, C.J., 1990. Differences in coordination of elbow flexor muscles in force tasks and in movement tasks. Exp. Brain Res. 81, 567572. https://doi.org/10.1007/BF02423505

Thelen, D.G., Schultz, A.B., Fassois, S.D., Ashton-Miller, J.A., 1994. Identification of dynamic myoelectric signal-to-force models during isometric lumbar muscle contractions. J. Biomech. 27, 907-919. https://doi.org/10.1016/0021-9290(94)90263-1

van den Bogert, A.J., Read, L., Nigg, B.M., 1999. An analysis of hip joint loading during walking, running, and skiing. Med Sci Sports Exerc 31, 131-42.

Wesseling, M., Groote, F.D., Bosmans, L., Bartels, W., Meyer, C., Desloovere, K., Jonkers, I., 2016. Subject-specific geometrical detail rather than cost function formulation affects hip loading calculation. Comput. Methods Biomech. Biomed. Engin. 0, 1-14. https://doi.org/10.1080/10255842.2016.1154547 
Figure 1: $\mathrm{R}^{2}$ and RMSE for hip flexion/extension moments for each calibration (unCal, minMoments, and minHJCF) and neural solution mode (EMG-driven, EMG-hybrid, EMGassisted, and static optimisation). Calibrated models produced higher $\mathrm{R}^{2}$ than the uncalibrated models.

Figure 2: The standard deviation $( \pm 1)$ curve for hip flexion/extension moments and hip joint contact forces for each neural solution mode (EMG-driven, EMG-hybrid, EMG-assisted, and static optimisation) and calibration (unCal, minMoments, and minHJCF). Hip flexion/extension moments are shown with hip flexion/extension moments calculated with inverse dynamics in OpenSim (Experimental). Hip joint contact forces are shown with hip joint contact forces recorded from instrumented hip implants (Bergmann et al., 2016).

Figure 3: Hip muscle excitation $\mathrm{R}^{2}$ and RMSE for EMG-assisted (top) and static optimisation (bottom). For each calibration (unCal, minMoments, and minHJCF), recorded hip muscle excitations were compared (GMED=gluteus medius, $\mathrm{GMAX}=$ gluteus maximus, $\mathrm{BFL}=$ biceps femoris long head, $\mathrm{SM}=$ semimembranosus, $\mathrm{AM}=$ adductor magnus, $\mathrm{RF}=$ rectus femoris, $\mathrm{SAR}=$ sartorius, $\mathrm{TFL}=$ tensor fascia latae, $\mathrm{GRA}=$ gracilis). EMG-assisted mode produced higher $\mathrm{R}^{2}$ and lower RMSE than static optimisation for all calibrations.
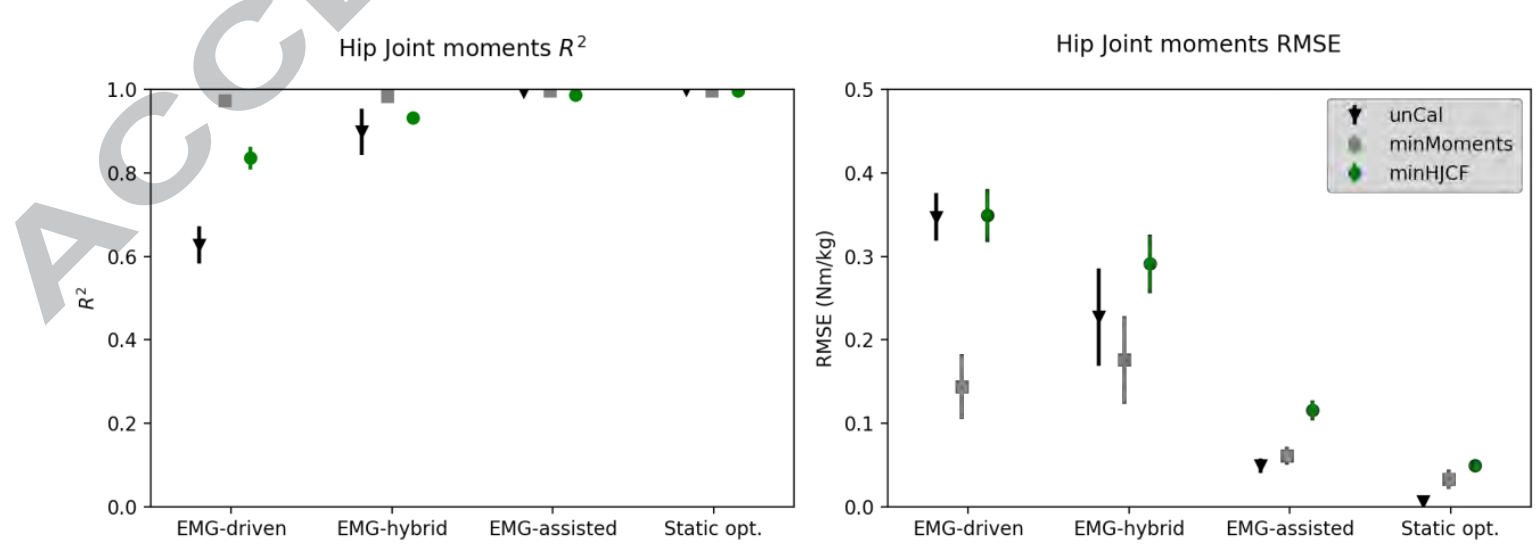

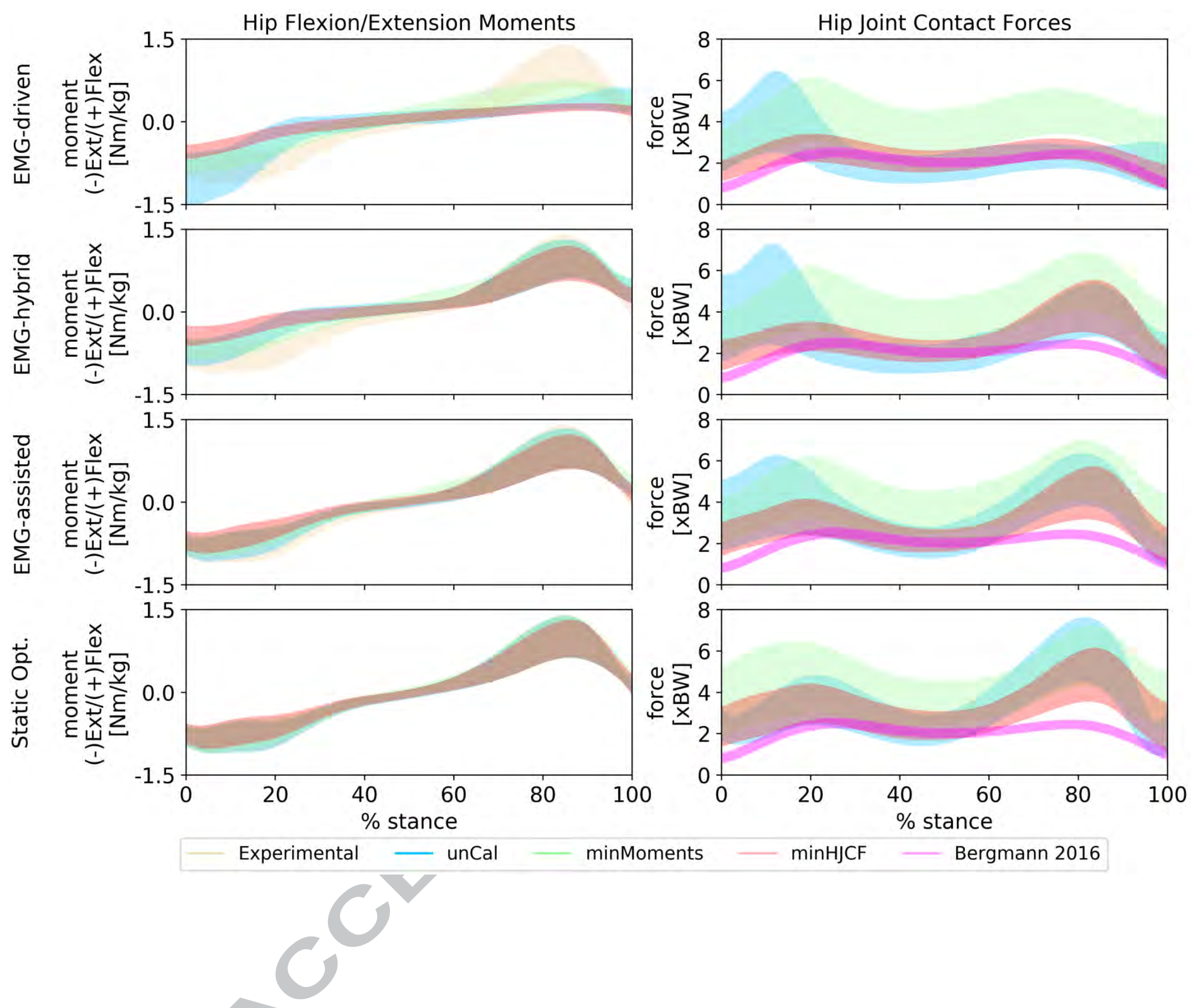

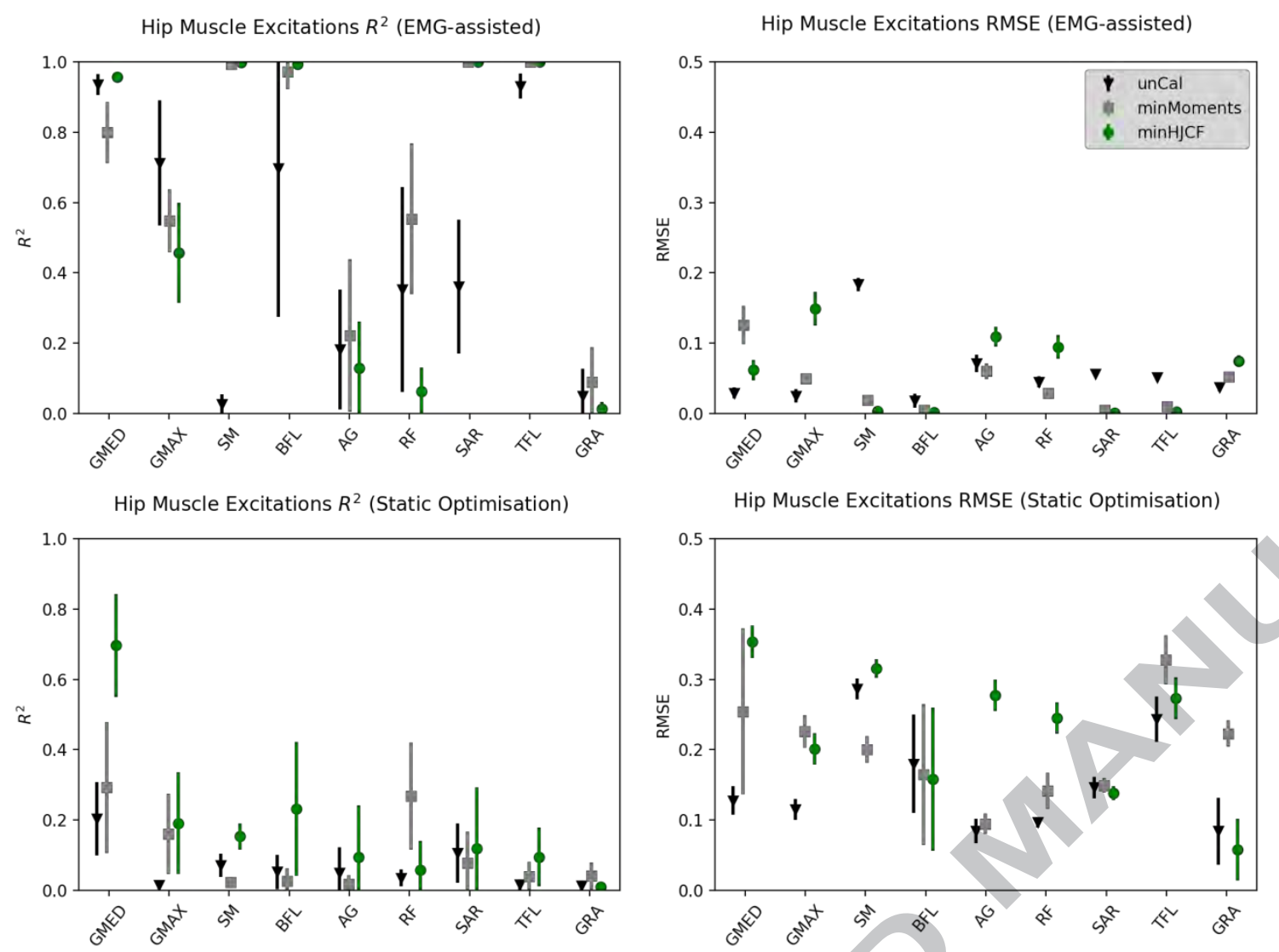

Table 1: Initial value and the boundary for the neuromuscular parameters adjusted during calibration for each muscle 


\begin{tabular}{|c|c|c|}
\hline Neuromuscular Parameter & Initial Value & Boundary \\
\hline Tendon slack length & Gait2392 value $^{a}$ & $\pm 15 \%$ of initial value \\
\hline Optimal fibre length ${ }^{b}$ & Gait2392 value $^{a}$ & $\pm 15 \%$ of initial value \\
\hline Maximum isometric force ${ }^{c}$ & Gait2392 value & $50 \%$ to $250 \%$ of initial value \\
\hline Non-linear shape factor $(A)$ & -0.1 & $(-3,0)$ \\
\hline $\begin{array}{l}\text { EMG-to-activation recursive filter } \\
\text { coefficients }\left(C_{1} \text { and } C_{2}\right)\end{array}$ & -0.5 & $(-0.95,-0.05)$ \\
\hline $\begin{array}{l}{ }^{a} \text { Before calibration, values wer } \\
\text { Modenese et al. (2016) (s } \\
{ }^{\mathrm{b}} \text { Normalised fibre length constr } \\
{ }^{\mathrm{c} S t r e n g t h ~ c o e f f i c i e n t ~ u s e d ~ t o ~ s c ~}\end{array}$ & $\begin{array}{l}\text { norphometrically } \\
\text { cription in text) } \\
(0.8,1.2) \\
\text { maximum isomet }\end{array}$ & $\begin{array}{l}\text { owing the procedure describe } \\
\text { loyd and Besier, 2003) }\end{array}$ \\
\hline
\end{tabular}

Table 2: $\mathrm{R}^{2}$ and RMSE joint moments for hip flexion/extension (FE), hip adduction/abduction (AA), hip internal/external rotation (IER), knee flexion/extension (FE), and ankle dorsi/plantar flexion (DPF) with standard deviation (STD) for each calibration (unCal, minMoments, minHJCF) and neural solution mode (EMG-driven, EMG-hybrid, EMG-assisted, Static Optimisation)

\begin{tabular}{|c|c|c|c|c|c|c|c|c|c|}
\hline & \multirow[b]{2}{*}{ calibration } & \multicolumn{2}{|c|}{ EMG-driven } & \multicolumn{2}{|c|}{ EMG-hybrid } & \multicolumn{2}{|c|}{ EMG-assisted } & \multicolumn{2}{|c|}{ Static Optimisation } \\
\hline & & $\mathrm{R}^{2} \pm S T D$ & $\begin{array}{c}\text { RMSE } \pm \text { STD } \\
\text { (Nm/kg) }\end{array}$ & $\mathrm{R}^{2} \pm S T D$ & $\begin{array}{c}\text { RMSE } \pm S T D \\
(\mathrm{Nm} / \mathrm{kg})\end{array}$ & $R^{2} \pm S T D$ & $\begin{array}{c}\text { RMSE } \pm S T D \\
(\mathrm{Nm} / \mathrm{kg})\end{array}$ & $R^{2} \pm S T D$ & $\begin{array}{c}\text { RMSE } \pm S T D \\
(\mathrm{Nm} / \mathrm{kg})\end{array}$ \\
\hline hip & unCal & $0.63 \pm 0.04$ & $0.35 \pm 0.03$ & $0.89 \pm 0.05$ & $0.23 \pm 0.05$ & $0.99 \pm 0.01$ & $0.05 \pm 0.01$ & $0.99 \pm 0.01$ & $0.01 \pm 0.01$ \\
\hline \multirow[t]{2}{*}{ FE } & minMoments & $0.96 \pm 0.01$ & $0.14 \pm 0.04$ & $0.98 \pm 0.01$ & $0.18 \pm 0.05$ & $0.99 \pm 0.01$ & $0.06 \pm 0.01$ & $0.99 \pm 0.01$ & $0.03 \pm 0.01$ \\
\hline & $\operatorname{minHJCF}$ & $0.85 \pm 0.03$ & $0.34 \pm 0.03$ & $0.93 \pm 0.01$ & $0.28 \pm 0.04$ & $0.99 \pm 0.01$ & $0.12 \pm 0.01$ & $0.99 \pm 0.01$ & $0.05 \pm 0.01$ \\
\hline hip & unCal & $0.23 \pm 0.10$ & $0.45 \pm 0.02$ & $0.27 \pm 0.11$ & $0.45 \pm 0.02$ & $0.93 \pm 0.01$ & $0.17 \pm 0.01$ & $0.99 \pm 0.01$ & $0.01 \pm 0.01$ \\
\hline $\mathbf{A A}$ & minMoments & $0.87 \pm 0.04$ & $0.13 \pm 0.02$ & $0.88 \pm 0.04$ & $0.12 \pm 0.02$ & $0.97 \pm 0.02$ & $0.07 \pm 0.02$ & $0.99 \pm 0.01$ & $0.04 \pm 0.01$ \\
\hline
\end{tabular}




\begin{tabular}{llllllcccc} 
& minHJCF & $0.83 \pm 0.04$ & $0.28 \pm 0.02$ & $0.83 \pm 0.04$ & $0.29 \pm 0.02$ & $0.95 \pm 0.02$ & $0.23 \pm 0.02$ & $0.92 \pm 0.01$ & $0.17 \pm 0.01$ \\
\hline hip & unCal & $0.58 \pm 0.05$ & $0.16 \pm 0.02$ & $0.54 \pm 0.05$ & $0.17 \pm 0.02$ & $0.92 \pm 0.02$ & $0.06 \pm 0.01$ & $0.99 \pm 0.01$ & $0.01 \pm 0.01$ \\
IER & minMoments & $0.85 \pm 0.03$ & $0.18 \pm 0.01$ & $0.84 \pm 0.03$ & $0.19 \pm 0.01$ & $0.89 \pm 0.04$ & $0.14 \pm 0.01$ & $0.87 \pm 0.03$ & $0.05 \pm 0.01$ \\
& minHJCF & $0.81 \pm 0.03$ & $0.07 \pm 0.01$ & $0.83 \pm 0.03$ & $0.06 \pm 0.01$ & $0.82 \pm 0.04$ & $0.05 \pm 0.01$ & $0.87 \pm 0.03$ & $0.06 \pm 0.01$ \\
\hline knee & unCal & $0.25 \pm 0.08$ & $0.34 \pm 0.03$ & $0.25 \pm 0.08$ & $0.34 \pm 0.03$ & $0.96 \pm 0.01$ & $0.06 \pm 0.01$ & $0.99 \pm 0.01$ & $0.01 \pm 0.01$ \\
FE & minMoments & $0.65 \pm 0.05$ & $0.13 \pm 0.02$ & $0.65 \pm 0.05$ & $0.13 \pm 0.02$ & $0.97 \pm 0.02$ & $0.05 \pm 0.01$ & $0.97 \pm 0.00$ & $0.04 \pm 0.01$ \\
& minHJCF & $0.62 \pm 0.06$ & $0.14 \pm 0.02$ & $0.62 \pm 0.06$ & $0.14 \pm 0.02$ & $0.97 \pm 0.02$ & $0.05 \pm 0.01$ & $0.98 \pm 0.01$ & $0.04 \pm 0.01$ \\
\hline ankle & unCal & $0.37 \pm 0.07$ & $0.37 \pm 0.02$ & $0.37 \pm 0.07$ & $0.37 \pm 0.02$ & $0.88 \pm 0.02$ & $0.17 \pm 0.02$ & $0.99 \pm 0.01$ & $0.01 \pm 0.01$ \\
DPF & minMoments & $0.89 \pm 0.03$ & $0.16 \pm 0.03$ & $0.89 \pm 0.04$ & $0.16 \pm 0.03$ & $0.98 \pm 0.01$ & $0.05 \pm 0.03$ & $0.97 \pm 0.02$ & $0.08 \pm 0.04$ \\
& minHJCF & $0.90 \pm 0.04$ & $0.15 \pm 0.03$ & $0.90 \pm 0.04$ & $0.15 \pm 0.03$ & $0.99 \pm 0.01$ & $0.05 \pm 0.02$ & $0.98 \pm 0.02$ & $0.07 \pm 0.03$ \\
\hline
\end{tabular}

unCal = uncalibrated model

minMoments = calibration minimising predicted joint moment error cost function

minHJCF = calibration minimising predicted joint moment error plus peak hip joint contact force cost function

Table 3: Average first and second peak hip joint contact force (Peak1 and Peak2, respectively) normalised to body weight (BW) with standard deviation (STD) for each calibration (unCal, minMoments, minHJCF) and neural solution mode (EMG-driven, EMG-hybrid, EMG-assisted, Static Optimisation)

\begin{tabular}{|c|c|c|c|c|c|c|c|c|}
\hline \multirow[b]{2}{*}{ calibration } & \multicolumn{2}{|c|}{ EMG-driven } & \multicolumn{2}{|c|}{ EMG-hybrid } & \multicolumn{2}{|c|}{ EMG-assisted } & \multicolumn{2}{|c|}{ Static Optimisation } \\
\hline & $\begin{array}{c}\text { Peak1 } \pm \text { STD } \\
\text { (BW) }\end{array}$ & $\begin{array}{c}\text { Peak2 } \pm \text { STD } \\
\text { (BW) }\end{array}$ & $\begin{array}{c}\text { Peak1 } \pm \text { STD } \\
\text { (BW) }\end{array}$ & $\begin{array}{c}\text { Peak2 } \pm \text { STD } \\
\text { (BW) }\end{array}$ & $\begin{array}{c}\text { Peak1 } \pm \text { STD } \\
\text { (BW) }\end{array}$ & $\begin{array}{l}\text { Peak2 } \pm \text { STD } \\
\text { (BW) }\end{array}$ & $\begin{array}{c}\text { Peak1 } 1 \pm \text { STD } \\
\text { (BW) }\end{array}$ & $\begin{array}{c}\text { Peak2 } \pm \text { STD } \\
\text { (BW) }\end{array}$ \\
\hline unCal & $4.75 \pm 1.57$ & $2.56 \pm 0.53$ & 5.0 & $3.68 \pm 0.98$ & $4.71 \pm 1.55$ & $4.73 \pm 0.94$ & $3.86 \pm 0.49$ & $5.49 \pm 1.06$ \\
\hline minMoments & $4.82 \pm 0.80$ & $4.63 \pm 0.73$ & $4.88 \pm 0.81$ & $5.25 \pm 1.00$ & $4.98 \pm 0.78$ & $5.27 \pm 1.01$ & $5.09 \pm 1.19$ & $5.17 \pm 0.99$ \\
\hline $\operatorname{minHJCF}$ & $2.83 \pm 0.36$ & $2.73 \pm 0.28$ & $2.85 \pm 0.39$ & $3.70 \pm 0.91$ & $3.55 \pm 0.99$ & $3.97 \pm 0.89$ & $3.75 \pm 1.17$ & $4.19 \pm 0.84$ \\
\hline
\end{tabular}


unCal = uncalibrated model

minMoments = calibration minimising predicted joint moment error cost function

minHJCF = calibration minimising predicted joint moment error plus peak hip joint contact force cost function




Table 4: Significant differences for each calibration (unCal, minMoments, minHJCF) and neural solution mode (EMG-driven, EMG-hybrid, EMG-assisted, Static Optimisation) for the resultant hip joint contact forces (HJCF)

\begin{tabular}{|c|c|c|c|c|}
\hline & neural solution mode & comparec & calibration & $p$-value a \\
\hline \multirow{12}{*}{$\begin{array}{l}\text { HJCF } \\
\text { peak } 1\end{array}$} & \multirow{3}{*}{ EMG-driven } & unCal & minMoments & 1.000 \\
\hline & & unCal & $\min H J C F$ & $<0.001$ \\
\hline & & minMoments & $\operatorname{minHJCF}$ & $<0.001$ \\
\hline & \multirow{3}{*}{ EMG-hybrid } & unCal & minMoments & 1.000 \\
\hline & & unCal & $\operatorname{minHJCF}$ & .011 \\
\hline & & minMoments & $\operatorname{minHJCF}$ & $<0.001$ \\
\hline & \multirow{3}{*}{ EMG-assisted } & unCal & minMoments & 1.000 \\
\hline & & unCal & $\min \mathrm{HJCF}$ & $<0.001$ \\
\hline & & minMoments & $\min \mathrm{HJCF}$ & $<0.001$ \\
\hline & \multirow{3}{*}{ Static Optimisation } & unCal & minMoments & $<0.001$ \\
\hline & & unCal & $\operatorname{minHJCF}$ & 1.000 \\
\hline & & minMoments & $\operatorname{minHJCF}$ & $<0.001$ \\
\hline \multirow{12}{*}{$\begin{array}{l}\text { HJCF } \\
\text { peak } 2\end{array}$} & \multirow{3}{*}{ EMG-driven } & & minMoments & $<0.001$ \\
\hline & & unCal & $\operatorname{minHJCF}$ & .128 \\
\hline & & minMoments & $\min \mathrm{HJCF}$ & $<0.001$ \\
\hline & \multirow{6}{*}{ EMG-assisted } & unCal & minMoments & $<0.001$ \\
\hline & & unCal & $\operatorname{minHJCF}$ & .352 \\
\hline & & minMoments & $\operatorname{minHJCF}$ & $<0.001$ \\
\hline & & unCal & minMoments & $<0.001$ \\
\hline & & unCal & $\min \mathrm{HJCF}$ & $<0.001$ \\
\hline & & minMoments & $\operatorname{minHJCF}$ & $<0.001$ \\
\hline & \multirow{3}{*}{ Static Optimisation } & unCal & minMoments & .031 \\
\hline & & unCal & $\operatorname{minHJCF}$ & $<0.001$ \\
\hline & & minMoments & $\operatorname{minHJCF}$ & $<0.001$ \\
\hline \multicolumn{5}{|c|}{ a One-way repeated measures ANOVA with Bonferroni adjustment (bold indicates significant at $p<0.05$ ) } \\
\hline \multicolumn{5}{|c|}{ unCal $=$ uncalibrated model } \\
\hline \multicolumn{5}{|c|}{$\begin{array}{l}\text { minMoments = calibration minimising predicted joint moment error cost function } \\
\text { minHJCF }=\text { calibration minimising predicted joint moment error plus peak hip joint contact force cost function }\end{array}$} \\
\hline
\end{tabular}


Table 5: Significant differences for each calibration (unCal, minMoments, minHJCF) and neural solution mode (EMG-assisted and static optimisation) for the $\mathrm{R}^{2}$ hip muscle excitations

\begin{tabular}{|c|c|c|c|c|}
\hline \multirow{2}{*}{\begin{tabular}{|l|} 
muscle \\
gluteus medius
\end{tabular}} & \multirow{2}{*}{$\begin{array}{l}\text { neural solution mode } \\
\text { EMG-assisted }\end{array}$} & \multicolumn{2}{|c|}{ compared calibration } & \multirow{2}{*}{$\frac{\text { p-value }^{\text {a }}}{<0.001}$} \\
\hline & & unCal & minMoments & \\
\hline & & unCal & $\operatorname{minHJCF}$ & $<0.001$ \\
\hline & & minMoments & $\operatorname{minHJCF}$ & $<0.001$ \\
\hline & Static Optimisation & unCal & minMoments & $<0.001$ \\
\hline & & unCal & $\operatorname{minHJCF}$ & $<0.001$ \\
\hline & & minMoments & $\operatorname{minHJCF}$ & $<0.001$ \\
\hline \multirow[t]{6}{*}{ gluteus maximus } & EMG-assisted & unCal & minMoments & $<0.001$ \\
\hline & & unCal & $\operatorname{minHJCF}$ & $<0.001$ \\
\hline & & minMoments & $\operatorname{minHJCF}$ & $<0.001$ \\
\hline & Static Optimisation & unCal & minMoments & $<0.001$ \\
\hline & & unCal & $\operatorname{minHJCF}$ & $<0.001$ \\
\hline & & minMoments & $\operatorname{minHJCF}$ & $<0.001$ \\
\hline \multirow[t]{6}{*}{ lateral hamstring group } & EMG-assisted & unCal & minMoments & $<0.001$ \\
\hline & & unCal & $\operatorname{minHJCF}$ & $<0.001$ \\
\hline & & minMoments & $\operatorname{minHJCF}$ & $<0.001$ \\
\hline & Static Optimisation & unCal & minMoments & $<0.001$ \\
\hline & & unCal & $\operatorname{minHJCF}$ & $<0.001$ \\
\hline & & minMoments & $\operatorname{minHJCF}$ & $<0.001$ \\
\hline \multirow[t]{6}{*}{ medial hamstring group } & EMG-assisted & unCal & minMoments & $<0.001$ \\
\hline & & unCal & $\operatorname{minHJCF}$ & $<0.001$ \\
\hline & & minMoments & $\operatorname{minHJCF}$ & 0.066 \\
\hline & Static Optimisation & unCal & minMoments & 0.836 \\
\hline & & unCal & $\operatorname{minHJCF}$ & 0.598 \\
\hline & & minMoments & $\operatorname{minHJCF}$ & 1.185 \\
\hline \multirow[t]{6}{*}{ adductor group } & EMG-assisted & unCal & minMoments & 0.021 \\
\hline & & unCal & $\operatorname{minHJCF}$ & $<0.001$ \\
\hline & & minMoments & $\operatorname{minHJCF}$ & $<0.001$ \\
\hline & Static Optimi & unCal & minMoments & 0.140 \\
\hline & & unCal & $\operatorname{minHJCF}$ & $<0.001$ \\
\hline & & minMoments & $\operatorname{minHJCF}$ & $<0.001$ \\
\hline \multirow[t]{6}{*}{ rectus femoris } & EMG-assisted & unCal & minMoments & $<0.001$ \\
\hline & & unCal & $\operatorname{minHJCF}$ & $<0.001$ \\
\hline & & minMoments & $\operatorname{minHJCF}$ & $<0.001$ \\
\hline & Static Optimisation & unCal & minMoments & $<0.001$ \\
\hline & & unCal & $\operatorname{minHJCF}$ & $<0.001$ \\
\hline & & minMoments & $\operatorname{minHJCF}$ & $<0.001$ \\
\hline \multirow[t]{6}{*}{ sartorius } & EMG-assisted & unCal & minMoments & $<0.001$ \\
\hline & & unCal & $\operatorname{minHJCF}$ & $<0.001$ \\
\hline & & minMoments & $\operatorname{minHJCF}$ & $<0.001$ \\
\hline & Static Optimisation & unCal & minMoments & 0.543 \\
\hline & & unCal & $\operatorname{minHJCF}$ & 0.051 \\
\hline & & minMoments & $\operatorname{minHJCF}$ & $<0.001$ \\
\hline \multirow[t]{6}{*}{ tensor fascia latae } & EMG-assisted & unCal & minMoments & $<0.001$ \\
\hline & & unCal & $\operatorname{minHJCF}$ & $<0.001$ \\
\hline & & minMoments & $\operatorname{minHJCF}$ & $<0.001$ \\
\hline & Static Optimisation & unCal & minMoments & $<0.001$ \\
\hline & & unCal & $\operatorname{minHJCF}$ & $<0.001$ \\
\hline & & minMoments & $\operatorname{minHJCF}$ & $<0.001$ \\
\hline \multirow[t]{6}{*}{ gracilis } & EMG-assisted & unCal & minMoments & $<0.001$ \\
\hline & & unCal & $\operatorname{minHJCF}$ & $<0.001$ \\
\hline & & minMoments & $\operatorname{minHJCF}$ & $<0.001$ \\
\hline & Static Optimisation & unCal & minMoments & $<0.001$ \\
\hline & & unCal & $\operatorname{minHJCF}$ & 0.088 \\
\hline & & minMoments & $\operatorname{minHJCF}$ & $<0.001$ \\
\hline \multicolumn{5}{|c|}{${ }^{a}$ One-way repeated measures ANOVA with Bonferroni adjustment (bold indicates significant at $p<0.05$ ) } \\
\hline \multicolumn{5}{|c|}{ unCal = uncalibrated model } \\
\hline \multicolumn{5}{|c|}{ minMoments = calibration minimising predicted joint moment error cost function } \\
\hline minHJCF = calibration mi & sing predicted joint moment & ror plus peak hip & int contac & functi \\
\hline
\end{tabular}




\section{Accepted Manuscript}

Subject-specific calibration of neuromuscular parameters enables neuromusculoskeletal models to estimate physiologically plausible hip joint contact forces

in healthy adults

Hoa X. Hoang, Claudio Pizzolato, Laura E. Diamond, David G. Lloyd

PII:

S0021-9290(18)30705-X

DOI:

https://doi.org/10.1016/j.jbiomech.2018.08.023

Reference:

BM 8834

To appear in:

Journal of Biomechanics

Accepted Date:

22 August 2018

Please cite this article as: H.X. Hoang, C. Pizzolato, L.E. Diamond, D.G. Lloyd, Subject-specific calibration of neuromuscular parameters enables neuromusculoskeletal models to estimate physiologically plausible hip joint contact forces in healthy adults, Journal of Biomechanics (2018), doi: https://doi.org/10.1016/j.jbiomech. 2018.08.023

This is a PDF file of an unedited manuscript that has been accepted for publication. As a service to our customers we are providing this early version of the manuscript. The manuscript will undergo copyediting, typesetting, and review of the resulting proof before it is published in its final form. Please note that during the production process errors may be discovered which could affect the content, and all legal disclaimers that apply to the journal pertain. 